\title{
Differential sensitivity to nicotine among hypothalamic magnocellular neurons
}

\author{
${ }^{1}$ Mikkelsen JD, ${ }^{1}$ Jacobsen J, ${ }^{2}$ Kiss A \\ ${ }^{1}$ Neurobiology Research Unit, Copenhagen University Hospital, Rigshospitalet, Denmark; ${ }^{2}$ Institute of Experimental \\ Endocrinology, Slovak Academy of Science, Bratislava, Slovakia \\ e-mail: jens_mikkelsen@dadlnet.dk
}

\begin{abstract}
Objectives. The magnocellular neurons in the hypothalamic paraventricular (PVN) and supraoptic nuclei (SON) either contain vasopressin or oxytocin. Even though both hormones are released after systemic administration of nicotine, the mechanism through which the two populations of neurons are activated is not known. This study was carried out in the rat to investigate the effect of increasing doses of nicotine on subsets of magnocellular neurons containing either oxytocin or vasopressin.

Methods. The activated neurons were identified by means of Fos immunohistochemistry and the induction of Fos in magnocellular subdivisions was investigated by means of dual-immunohistochemistry.

Results. While oxytocinergic neurons were sensitive to systemic administration of $0.5 \mathrm{mg} / \mathrm{kg}$ of nicotine, vasopressinergic neurons were not affected at doses up to $1 \mathrm{mg} / \mathrm{kg}$. The vast majority (85\%) of oxytocinergic neurons in the PVN was affected by nicotine, whilst only about half of the vasopressinergic neurons were stimulated, and only at maximal doses. Notably, the sensitivity of oxytocinergic neurons to nicotine was found to be different in the PVN and SON, because only about $55 \%$ of the SON oxytocinergic neurons co-stored Fos even after the highest dose of nicotine.

Conclusion. These data show that magnocellular neurons are differentially regulated by nicotine and that their sensitivity is dependent on both their peptidergic phenotype and their location within the hypothalamus.
\end{abstract}

Key words: acetylcholine, vasopressin, oxytocin, Fos, stress, cell counting

Neurons in the PVN and SON projecting to the posterior lobe of the pituitary synthesise either vasopressin or oxytocin and secrete these two hormones into the general circulation. The magnocellular neurons in the PVN and SON have similar morphology and are considered to share functional properties. Release of one or both of these neuropeptides occurs under a variety of physiological conditions including dehydration, parturition, and lactation. Such diverse behaviours associate to different neural systems and pathways that interact with magnocellular neurons. It is therefore evident that the organisation and excitability of inputs to the magnocel- lular neurons are orchestrated to produce appropriate hormonal outputs to external and pharmacological stimuli (Hatton 1997). Magnocellular vasopressinergic and oxytocinergic neurons are distinct and receive different inputs. Observations of functional differences among neurons within the hypothalamus that express the same neuropeptide are more controversial, since they are considered to share inputs and neurophysiological characteristics (Hatton 1997).

The magnocellular neurons of the PVN that contain oxytocin are located in the anterior and medial magnocellular groups, while vasopressinergic neurons are present 
in the main body of the posterior magnocellular group (Armstrong 1995). The two populations are characterised by a distinct firing pattern that emerges when the cells are activated (Li et al. 1995). While oxytocinergic neurons increase their firing rate and reduce interspike intervals, vasopressinergic neurons display prolonged bursts of action potentials with long silent periods. In addition, there is an oxytocinergic parvocellular subnucleus in the dorsal PVN that sends descending projections to brain centres mainly involved in the central autonomic control such as the periaquaductal grey matter, parabrachial nucleus, nucleus of the solitary tract, rostral ventrolateral reticular nucleus, and to both parasympathetic and sympathetic preganglionic neurons in the medulla oblongata and spinal cord (Saper et al. 1976; Luiten et al. 1985; Hallbeck et al. 2001). There is also a population of parvocellular vasopressinergic neurons that co-store corticotrophin releasing hormone $(\mathrm{CRH})$ and is involved in the regulation of the hypothalamo-pituitary adrenal (HPA) axis (Harbuz and Lightman 1992).

Acetylcholine (ACh) is one of several neurotransmitters involved in the regulation of hypothalamic outflow, and this effect is primarily mediated through nicotinic ACh receptors (nAChR). Systemic administration of nicotine in animals and cigarette smoking in man increase plasma concentrations of vasopressin and oxytocin (Andersson et al. 1983; Chiodera et al. 1993; Rhodes et al. 2001). In addition, nicotine increases intracellular $\mathrm{Ca}^{2+}$ concentration and vasopressin gene expression in the magnocellular neurons of the SON in vitro (Shioda et al. 1997a; Shioda et al. 1997b). Although the HPA axis is strongly stimulated by systemic administration of nicotine (Rhodes et al. 2001), the parvocellular neurons are insensitive to $\mathrm{ACh}$ in vitro (Zaninetti et al. 2002) suggesting that these cells are activated through an indirect pathway. One pathway could involve catecholaminergic afferents in the PVN, originating in the medulla oblongata, because these are also stimulated by nicotine (Matta et al. 1993; Hatton 1997). In addition, the hypocretinergic neurons in the lateral hypothalamus may play a role (Plaza-Zabala et al. 2012).

With regard to the magnocellular neurons, much less is known about the pathways and mechanisms through which nicotine affects them. This study was carried out to determine whether magnocellular neurons are stimulated by systemic administration of nicotine in terms of increased synthesis of the transcription factor Fos. Dual-immunohistochemistry was conducted to reveal if the sensitivity in PVN or SON magnocellular neurons to nicotine was dependent on their neuropeptidergic phenotype.

\section{Materials and methods}

Animals and drug treatments. Male Wistar rats (Taconic, Ll Skensved, Denmark) weighing 200-250 grams were used in this study. The rats had free access to food and water, and were adjusted to the new animal facility for at least two weeks upon arrival. The animals were kept in the experimental room at least one the day before the experiment. At the experimental day, the animals were divided into groups and each were injected with nicotine $(0.5,1.0$, or $2.0 \mathrm{mg} / \mathrm{kg}$ s.c.; (-)- nicotine-dihydrogentartrat, Sigma-Aldrich, Free base) or vehicle at $4 \mathrm{~h}$ after the onset of the light phase and returned to their home cage. Two hours after the injection the animals were deeply anaesthetized with mebumal and perfused transcardially with $0.9 \%$ saline followed by $4 \%$ paraformaldehyde in $0.1 \mathrm{M}$ phosphate buffer ( $\mathrm{pH} \mathrm{7.4)}$ for $10 \mathrm{~min}$. The brains were post-fixed overnight and subsequently submerged in $30 \%$ sucrose in $0.01 \mathrm{M}$ phosphate-buffered ( $\mathrm{pH} 7.4$ ) saline (PBS) at 4 ${ }^{\circ} \mathrm{C}$ for 3 days. Forty $\mu \mathrm{m}$ serial coronal sections were cut on a freezing microtome in series of six and subjected to single and dual-immunohistochemistry.

Immunohistochemistry. Prior to the single immunohistochemical steps, the sections were rinsed for $3 \mathrm{x}$ $15 \mathrm{~min}$ in $0.05 \mathrm{M}$ PBS. Before incubation in the primary antiserum, the sections were incubated in $1 \% \mathrm{H}_{2} \mathrm{O}_{2}-\mathrm{PBS}$ for $10 \mathrm{~min}$, and in PBS with $0.3 \%$ Triton X-100 and 1\% bovine serum albumin (BSA) for $30 \mathrm{~min}$. The sections were then incubated at $4{ }^{\circ} \mathrm{C}$ for $24 \mathrm{~h}$ in primary rabbit antiserum against Fos (rabbit \#94012) diluted 1:4,000 as characterised earlier (Woldbye et al. 1996; Mikkelsen et al. 1998). The sections were washed for $3 \times 10 \mathrm{~min}$ in washing buffer (PBS $+0.1 \%$ Triton X-100, and $0.3 \% \mathrm{BSA}$ ), and incubated for $60 \mathrm{~min}$ in biotinylated donkey anti-rabbit serum (Jackson ImmunoResearch Laboratories, Inc. USA, diluted 1:1000) in the same buffer, washed for $3 \times 10 \mathrm{~min}$ and incubated for $60 \mathrm{~min}$ in an avidin-biotin complex (VectorStain Elite $\mathrm{ABC}$ Kit, Vector Lab., Burlingame, CA, USA). The sections were carefully washed and incubated for $10 \mathrm{~min}$ in $0.2 \% \mathrm{w} / \mathrm{v} \mathrm{NiSO}$ in $0.1 \mathrm{M} \mathrm{Na}$-acetate $\mathrm{pH} 6.0$ followed by incubation in the same buffer $+0.025 \% \mathrm{DAB}+0.05 \%$ $\mathrm{H}_{2} \mathrm{O}_{2}$ for $6 \mathrm{~min}$. Then the sections were washed twice in PBS. The un-reacted peroxidase was blocked by incubation in $1.5 \% \mathrm{H}_{2} \mathrm{O}_{2}$ in PBS for $15 \mathrm{~min}$. The brains were processed for simultaneous detection of vasopressin or 
oxytocin and Fos in the same tissue sections by repeating the procedure above after incubation with either rabbit anti-vasopressin (1:25K; Bachem, Weilan Rhein, Germany) or rabbit anti-oxytocin sera (1:10K; Millipore, Billerica, USA). Finally, after a careful wash they were incubated in $0.05 \% \mathrm{DAB}+0.05 \% \mathrm{H}_{2} \mathrm{O}_{2}$ in $\mathrm{PBS}$ for $7 \mathrm{~min}$ and then washed twice in $\mathrm{H}_{2} \mathrm{O}$, mounted on gelatinized glass slides, dried, and coverslipped in Depex ${ }^{\oplus}$.

Data analysis. Photoscans were taken directly on a Nikon microscope and the absolute number and percentages of dual labelled cells were counted manually in defined structures by an observer blinded to the treatment. The counting for Fos was performed on one section through the mid-portion of the PVN or SON. The percentage of dual-labeled cells was counted from two consecutive sections through the anterior, middle, and posterior subdivisions of the PVN and SON, and the average of relative numbers of dual-labeled cells for each animal was determined.

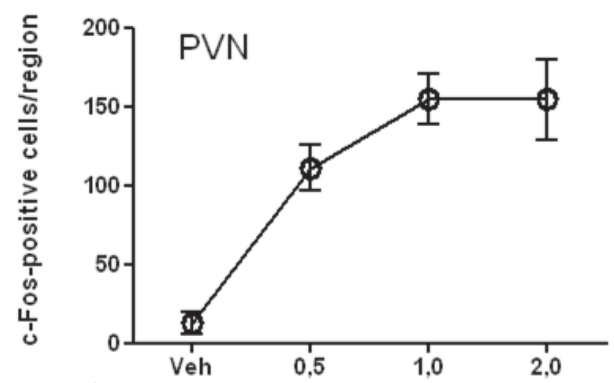

A

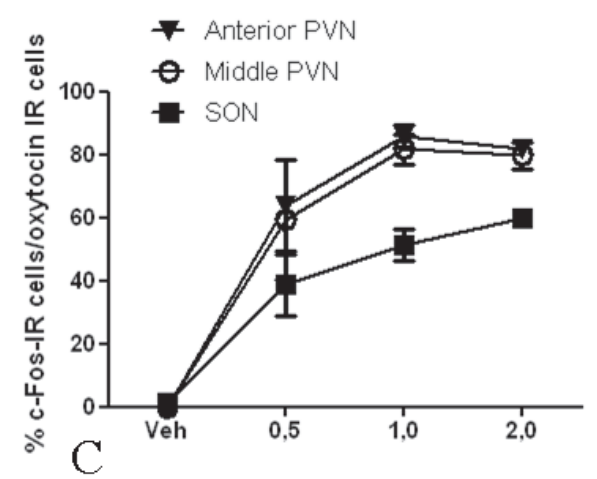

\section{Results}

The expression of Fos in rats treated systemically with increasing concentrations of nicotine was analyzed in the SON, the PVN subnuclei as well as in the islands of magnocellular neurons within the hypothalamus, but situated outside the PVN and SON. In saline-treated rats, virtually no immunolabeled nuclei were found in the SON. Only a low to moderate number of weakly labeled nuclei were present mainly in the parvocellular division of the PVN in saline-treated animals. Total counts of immunolabeled nuclei revealed that nicotine produced a dose-dependent induction of Fos in both the PVN and SON (Figs 1A, 1B). The dose-response curves were different between the PVN and SON, and may illustrate a difference in sensitivity to nicotine. When determining the total number of labeled cells, higher dose of nicotine produced an increase in labeled neurons in the SON (Fig. 1B). In contrast, the number

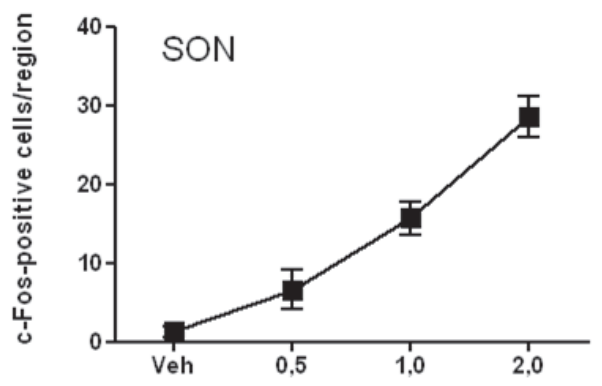

$\mathrm{B}$

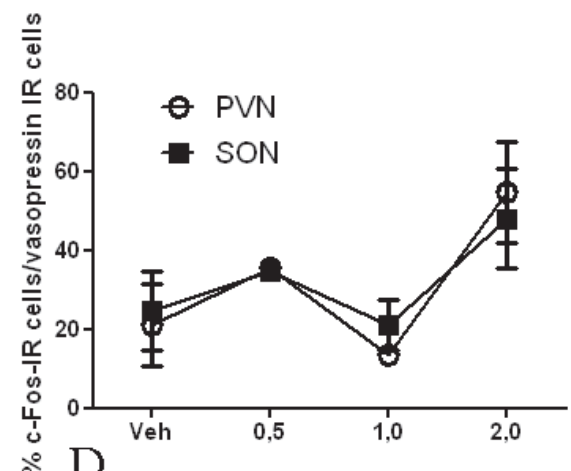

$\mathrm{D}$

Fig. 1. Dose-response curves showing the effects of increasing subcutaneaous doses $(\mathrm{mg} / \mathrm{kg})$ of nicotine on the total number of Fos positive nuclei in the PVN (A) and SON (B). Figs C and D illustrate the relative number of Fos containing oxytocinergic (C) and vasopressinergic neurons (D) in various subdivisions of the magnocellular nuclei in the same animals. It should be noted that the relative number of oxytocinergic neurons in the anterior and middle PVN was $85 \%$ whereas the number of oxytocinergic neurons in the SON did not exceed 55\% (C) $(\mathrm{P}<0,01$, two way ANOVA). 


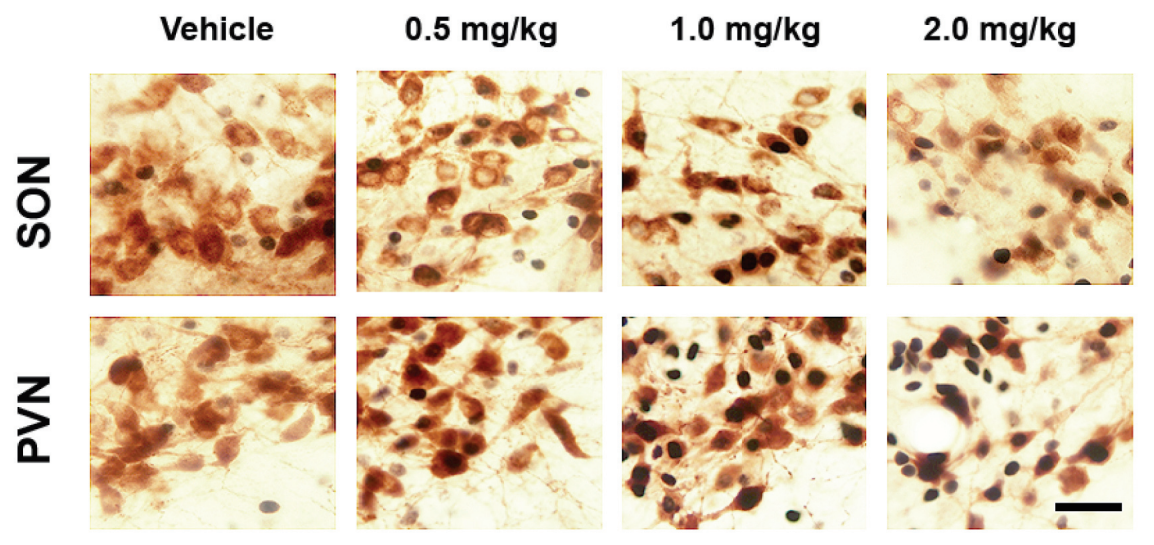

Fig. 2. Illustration of dual-labeled neurons for Fos and oxytocin immunoreactivities in animals exposed to increasing concentrations of systemic nicotine. Images of the SON are shown in the upper row and PVN in the lower row. Scale bar $=100 \mu \mathrm{m}$

of Fos-positive neurons in the PVN reached a maximum after nicotine in doses of $1 \mathrm{mg} / \mathrm{kg}$. In the magnocellular portions of the PVN the Fos positive nuclei occupied the anterior, lateral, and posterior magnocellular subnuclei, and the medial parvocellular subnucleus.

Concurrent dual-immunohistochemistry was performed on tissue from the same animals in an effort to determine the extent of Fos induction in vasopressinergic and oxytocinergic neurons, respectively. An extensive overlap of nuclear Fos immunolabeling in oxytocin-positive neurons was observed (Figs. 1C, 2 , 3A-B). About $60 \%$ of the oxytocin neurons in the anterior and middle magnocellular PVN co-stored Fos after nicotine at doses of $0.5 \mathrm{mg} / \mathrm{kg}$, and the number of co-labeled neurons increased to about $85 \%$ at higher concentrations (Figs. 1C, 2, and 3A). The proportion of double-labeled neurons in the anterior and middle magnocellular subdivisions of the PVN was the same (Fig. 1C). The sensitivity of oxytocinergic neurons in the SON was similar to the PVN as only a minor increase in dual-labeled neurons between a dose of $0.5 \mathrm{mg} / \mathrm{kg}$ and $1.0 \mathrm{mg} / \mathrm{kg}$ was detected (Figs. 1C and 2). Notably, the proportion of co-labeled neurons in the SON did not exceed more than $55 \%$ even at a maximal dose of nicotine $(2.0 \mathrm{mg} / \mathrm{kg})$ (Figs. $1 \mathrm{C}$ and 2$)$. The islands of magnocellular neurons distributed in small groups throughout the hypothalamus and not confined to either the PVN or SON (Armstrong 1995) was also extensively filled with nuclear Fos. There was a tendency that islands located in proximity to the SON contained less double labeled neurons than those located closer to the PVN. No co-labeling after nicotine was observed in the oxytocinergic neurons in the posterior parvocellular PVN (Fig. 3C).

\section{PVN}
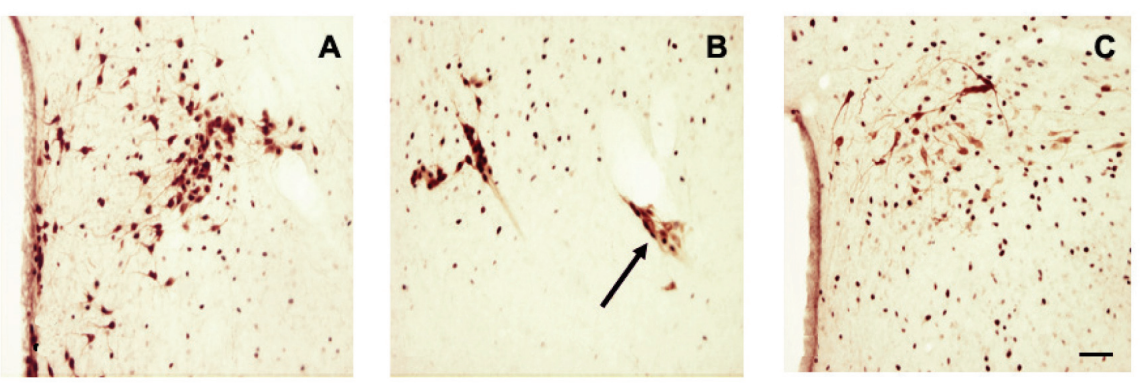

Fig. 3. Dual-labeling of Fos and oxytocin in the anterior subpart of the PVN (Fig. 3A), islands of the basomedial hypothalamus (Fig. 3B) and the dorsal posterior parvocellular subnucleus of the PVN (Fig. 3C). The number of dual-labeled neurons in the cell group to the right (arrow) that is situated close to the SON contains less dual-labeled neurons than the one to the left and located closer to the PVN. Scale bar $=100 \mu \mathrm{m}$ 


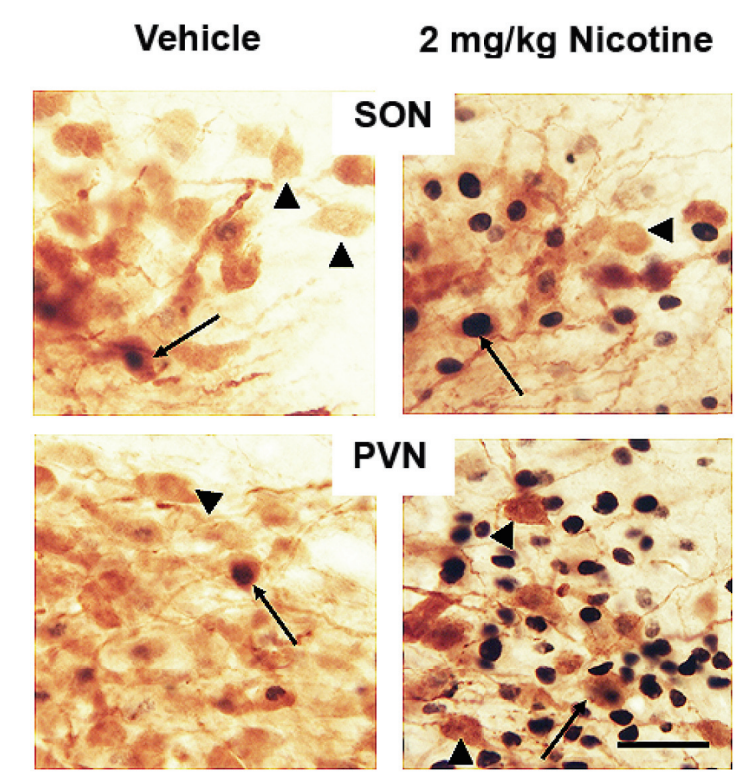

Fig 4. Illustration of dual-labeled neurons for Fos and vasopressin immunoreactivities in the SON and PVN. Arrow-heads point to single and arrows to dual-labeled neurons in each image. Scale bar $=50 \mu \mathrm{m}$

The effects of nicotine on vasopressinergic magnocellular neurons were different from the effect on oxytocinergic neurons. Firstly, a basal level of Fos in the magnocelular vasopressinergic neurons was higher (Figs. 1D and 4). Secondly, even though, the relative number of vasopressinergic neurons co-storing nuclear Fos in the posterior magnocellular subnucleus of the PVN or in the SON was slightly higher in animals treated with nicotine. However, it did not reach significance even at the highest dose of $2.0 \mathrm{mg} / \mathrm{kg}$.

\section{Discussion}

In the present study, nicotine was shown to produce a dose-dependent excitation of neurons within the hypothalamo-neurohypophysial system and the HPA axis in terms of activation of the AP-1 transcription factor, Fos. These results are in agreement with earlier observations, showing that systemic administration of nicotine increases plasma concentrations of oxytocin, vasopressin, prolactin, and ACTH (Andersson et al. 1983; Sharp and Beyer 1986; Rhodes et al. 2001).

There are two new findings in the present study. First, the sensitivity to nicotine in vivo was found to be different between magnocellular phenotypes. Second, some oxytocinergic neurons, most of which were located in the SON, were not activated by nicotine, suggesting that the activation of magnocellular neurons and release of their respective hormones are regulated at several levels.
A mechanism behind the observed diversity could be that different nAChR subtypes (Sargent 1993), that are differentially sensitive to systemic nicotine, activate distinct magnocellular neurons. Another possibility is that there exists a topographical organization of the different input systems displaying various sensitivities to nicotine.

Magnocellular neurons have in pharmacological studies been shown to bind $\alpha_{7}$ nACh specific agents and nicotine acts in vitro via postsynaptic $\alpha_{7} \mathrm{nACh}$ receptors located within the SON or in its immediate surroundings (Hatton and Yang 2002; Zaninetti et al. 2002). Patch clamp recordings have shown that the amplitude of ACh-evoked currents was similar in vasopressinergic and oxytocinergic neurons, suggesting that the two neuronal populations are equally sensitive to nicotine probably via their postsynaptic $\alpha_{7} \mathrm{nACh}$ receptors (Zaninetti et al. 2002).

Our data indicate that the effects of nicotine are mediated via different $n A C h R s$ receptors. Since functional nAChRs probably of the $\alpha_{7}$ nAChR subtype have been found in oxytocinergic neurons, the signaling mediated through this nAChR subtype may work independently of Fos activation in these neurons. Further, it is unlikely that $\alpha_{7} \mathrm{nAChR}$ expressed in the oxytocinergic neurons are necessary to produce induction in Fos in these cells. This is supported by the observation that nicotineinduced Fos in the PVN and SON cannot be blocked 
by the $a_{7} \mathrm{nAChR}$ antagonist methyllycaconitine (Seppa et al. 2001). Even though $\alpha_{7} \mathrm{nAChR}$ are expressed in magnocellular neurons, it is unlikely that postsynaptic activation of this receptor in these neurons lead to Fos induction. This is also supported by the observation that $\alpha_{7} \mathrm{nAChR}$ agonists only induce Fos in a restricted part of areas expressing $\alpha_{7} \mathrm{nAChR}$ binding (Hansen et al. 2007; Thomsen et al. 2008). Recent reports in other neuronal systems have shown that the $\alpha_{7} \mathrm{nAChR}$-dependent signaling pathway involves JAK2 binding to the receptor and subsequent phosphoinositol 3-kinase activation that phosphorylates Akt in vitro (Kihara et al. 2001; Shaw et al. 2002), but whether $\alpha_{7} \mathrm{nAChR}$ mediate this signaling pathway in the magnocellular neurons remains to be established.

As it is unlikely that Fos is induced as a result of direct activation of nicotinergic postsynaptic receptors in the oxytocinergic neurons, the induction may be a result of either release from presynaptic terminals near the magnocellular nuclei or from activation of afferent neurons. Fos induction in SON neurons requires synaptic activation (Luckman et al. 1994), which speak in favor of presynaptic activation in response to nicotine. The major afferent signals to the SON are those from excitatory glutamatergic inputs and inhibitory GABAergic inputs (Wuarin 1997). Nicotine applied to hypothalamic slices has been shown to increase the frequency of excitatory and suppressed inhibitory postsynaptic currents (Hallbeck et al. 2001). Part of the action may act via an activation of presynaptic $\alpha_{4} \beta_{2}$ receptors leading to a release of noradrenaline in the SON as seen after local application in the hypothalamus (Liu et al. 2001). Furthermore, a release of noradrenaline also occurs via stimulation of pathways originating in the catecholaminergic neurons in the brain stem that are also involved in the nicotine-dependent activation of the stress axis (Matta et al. 1993).
One striking observation was that more oxytocinergic neurons in the PVN responded to nicotine than in the SON. The reason why SON oxytocinergic neurons are less sensitive to nicotine-induced Fos activation is at present unclear. Even virtually all inputs to the SON also go to the PVN, one exception is the mitral cells of the olfactory bulb that innervate the SON, but not the PVN (Smithson et al. 1989). Such distinct input pattern may account for a distinction in the response to nicotine. Another tempting speculation is that the microenvironment in the PVN is different from the SON and may produce an increase in Fos. Thus, it is possible that release of neurotransmitters in the efferent system innervating the medial parvocellular part of $\mathrm{CRH}$ or vasopressin released by the parvocellular neurons, may elicit a response in the magnocellular neurons leading to expression of Fos. However, intracerebroventricular administration of CRH and vasopressin has not been reported to produce any induction of Fos in magnocellular neurons (Andreae and Herbert 1993; Bittencourt and Sawchenko 2000). Finally, nicotine may act differently in the two nuclei with respect to presynaptic action, i.e. release of noradrenaline or histamine (Hallbeck et al. 2001; Liu et al. 2001).

The PVN exerts many of its regulatory functions through projections to the spinal cord, and oxytocinergic neurons account for a large proportion of these descending pathways (Hallbeck et al. 2001). However, these neurons are not affected indicating that the characteristics of nicotine-induced Fos are not linked to the oxytocin phenotype as such, but rather the functional properties of input pathways.

\section{Acknowledgements}

This work was supported by the NOVO Nordisk Foundation, The Lundbeck Foundation, and the Danish Medical Research Council for Strategic Research.

\section{References}

Andersson K, Siegel R, Fuxe K, Eneroth P: Intravenous injections of nicotine induce very rapid and discrete reductions of hypothalamic catecholamine levels associated with increases of ACTH, vasopressin and prolactin secretion. Acta Physiol Scand 118, 35-40, 1983. http://dx.doi.org/10.1111/j.1748-1716.1983.tb07237.x

Andreae LC, Herbert J: Expression of c-fos in restricted areas of the basal forebrain and brainstem following single or combined intraventricular infusions of vasopressin and corticotropin-releasing factor. Neuroscience 53, 735-748, 1993. http://dx.doi.org/10.1016/0306-4522(93)90620-U

Armstrong WE: Hypothalamic supraoptic and paraventricular nuclei. In The rat brain, ed. Paxinos, pp. 377-390, 1995. 
Bittencourt JC, Sawchenko PE: Do centrally administered neuropeptides access cognate receptors?: an analysis in the central corticotropin-releasing factor system. J Neurosci 20, 1142-1156, 2000.

Chiodera P, Volpi R, Capretti L, Bocchi R, Caffarri G, Marcato A, Rossi G, Coiro V: Gamma-aminobutyric acid mediation of the inhibitory effect of endogenous opioids on the arginine vasopressin and oxytocin responses to nicotine from cigarette smoking. Metabolism 42:762-765, 1993. http://dx.doi.org/10.1016/0026-0495(93)90246-K

Hallbeck M, Larhammar D, Blomqvist A: Neuropeptide expression in rat paraventricular hypothalamic neurons that project to the spinal cord. J Comp Neurol 433, 222-238, 2001. http://dx.doi.org/10.1002/cne.1137

Hansen HH, Timmermann DB, Peters D, Walters C, Damaj MI, Mikkelsen JD: Alpha-7 nicotinic acetylcholine receptor agonists selectively activate limbic regions of the rat forebrain: an effect similar to antipsychotics. Journal of neuroscience research 85, 1810-1818, 2007. http://dx.doi.org/10.1002/jnr.21293

Harbuz MS, Lightman SL: Stress and the hypothalamo-pituitary-adrenal axis: acute, chronic and immunological activation. The Journal of endocrinology 134, 327-339, 1992. http://dx.doi.org/10.1677/joe.0.1340327

Hatton G:. Function-related plasticity in hypothalamus. Annu Rev Neurosci 20, 375-397, 1997. http://dx.doi.org/10.1146/ annurev.neuro.20.1.375

Hatton GI, Yang QZ: Synaptic potentials mediated by alpha 7 nicotinic acetylcholine receptors in supraoptic nucleus. J Neurosci 22, 29-37, 2002.

Kihara T, Shimohama S, Sawada H, Honda K, Nakamizo T, Shibasaki H, Kume T, Akaike A: Alpha 7 nicotinic receptor transduces signals to phosphatidylinositol 3-kinase to block A beta-amyloid-induced neurotoxicity. J Biol Chem 276, 13541-13546, 2001.

Li Z, Decavel C, Hatton GI: Calbindin-D28k: role in determining intrinsically generated firing patterns in rat supraoptic neurones. J Physiol 488 (Pt 3), 601-608, 1995.

Liu X, Onaka T, Yagi K: Nicotine facilitates noradrenaline release in the rat supraoptic nucleus. Neuroreport 12, 641-643, 2001. http://dx.doi.org/10.1097/00001756-200103050-00042

Luckman SM, Dyball RE, Leng G: Induction of c-fos expression in hypothalamic magnocellular neurons requires synaptic activation and not simply increased spike activity. J Neurosci 14, 4825-4830, 1994.

Luiten PG, ter Horst GJ, Karst H, Steffens AB: The course of paraventricular hypothalamic efferents to autonomic structures in medulla and spinal cord. Brain Res 329, 374-378, 1985. http://dx.doi.org/10.1016/0006-8993(85)90554-2

Matta SG, Foster CA, Sharp BM: Nicotine stimulates the expression of cFos protein in the parvocellular paraventricular nucleus and brainstem catecholaminergic regions. Endocrinology 132, 2149-2156, 1993. http://dx.doi.org/10.1210/ en.132.5.2149

Mikkelsen JD, Vrang N, Mrosovsky N: Expression of Fos in the circadian system following nonphotic stimulation. Brain Res Bull 47, 367-376, 1998. http://dx.doi.org/10.1016/S0361-9230(98)00121-X

Plaza-Zabala A, Flores A, Maldonado R, Berrendero F: Hypocretin/Orexin signaling in the hypothalamic paraventricular nucleus is essential for the expression of nicotine withdrawal. Biol psychiatry 71, 214-223, 2012. http://dx.doi. org/10.1016/j.biopsych.2011.06.025

Rhodes ME, O'Toole SM, Czambel RK, Rubin RT: Male-female differences in rat hypothalamic-pituitary-adrenal axis responses to nicotine stimulation. Brain Res Bull 54, 681-688, 2001. http://dx.doi.org/10.1016/S03619230(01)00488-9

Saper CB, Loewy AD, Swanson LW, Cowan WM: Direct hypothalamo-autonomic connections. Brain Res 117, 305-312, 1976. http://dx.doi.org/10.1016/0006-8993(76)90738-1

Sargent PB: The diversity of neuronal nicotinic acetylcholine receptors. Annu Rev Neurosci 16, 403-443, 1993. http:// dx.doi.org/10.1146/annurev.ne.16.030193.002155

Seppa T, Salminen O, Moed M, Ahtee L: Induction of Fos-immunostaining by nicotine and nicotinic receptor antagonists in rat brain. Neuropharmacology 41, 486-495, 2001. http://dx.doi.org/10.1016/S0028-3908(01)00093-4

Sharp BM, Beyer HS: Rapid desensitization of the acute stimulatory effects of nicotine on rat plasma adrenocorticotropin and prolactin. J Pharmacol Exp Ther 238, 486-491, 1986.

Shaw S, Bencherif M, Marrero MB: Janus kinase 2, an early target of alpha 7 nicotinic acetylcholine receptor-mediated neuroprotection against Abeta-(1-42) amyloid. J Biol Chem 277, 44920-44924, 2002. http://dx.doi.org/10.1074/ jbc.M204610200

Shioda S, Nakajo S, Hirabayashi T, Nakayama H, Nakaya K, Matsuda K, Nakai Y: Neuronal nicotinic acetylcholine receptor in the hypothalamus: morphological diversity and neuroendocrine regulations. Brain Res Mol Brain Res 49, 45-54, 1997a. http://dx.doi.org/10.1016/S0169-328X(97)00122-8

Shioda S, Yada T, Muroya S, Takigawa M, Nakai Y: Nicotine increases cytosolic Ca2+ in vasopressin neurons. Neurosci Res 29, 311-318, 1997b. http://dx.doi.org/10.1016/S0168-0102(97)00103-X 
Smithson KG, Weiss ML, Hatton GI: Supraoptic nucleus afferents from the main olfactory bulb--I. Anatomical evidence from anterograde and retrograde tracers in rat. Neuroscience 31, 277-287, 1989. http://dx.doi.org/10.1016/03064522(89)90373-4

Thomsen MS, Mikkelsen JD, Timmermann DB, Peters D, Hay-Schmidt A, Martens H, Hansen HH: The selective alpha7 nicotinic acetylcholine receptor agonist A-582941 activates immediate early genes in limbic regions of the forebrain: Differential effects in the juvenile and adult rat. Neuroscience 154, 741-753, 2008. http://dx.doi. org/10.1016/j.neuroscience.2008.03.083

Woldbye DP, Greisen MH, Bolwig TG, Larsen PJ, Mikkelsen JD: Prolonged induction of c-fos in neuropeptide Y- and somatostatin-immunoreactive neurons of the rat dentate gyrus after electroconvulsive stimulation. Brain Res 720, 111-119, 1996. http://dx.doi.org/10.1016/0006-8993(96)00158-8

Wuarin JP: Glutamate microstimulation of local inhibitory circuits in the supraoptic nucleus from rat hypothalamus slices. J Neurophysiol 78, 3180-3186, 1997.

Zaninetti M, Tribollet E, Bertrand D, Raggenbass M: Nicotinic cholinergic activation of magnocellular neurons of the hypothalamic paraventricular nucleus. Neuroscience 110, 287-299, 2002. http://dx.doi.org/10.1016/ $\underline{\text { S0306-4522(01)00536-X }}$ 